\title{
On some divisor problems
}

\author{
by
}

\section{HONG-QUAN LiU (Harbin)}

1. Introduction. We investigate the distribution of the divisor functions $d(1,1,2 ; n)$ and $d(1,1,2,2 ; n)$, which are defined as

$$
\begin{gathered}
d(1,1,2 ; n)=\#\left\{\left(n_{1}, n_{2}, n_{3}\right) \mid n_{1}, n_{2}, n_{3} \in \mathbb{N}, n_{1} n_{2} n_{3}^{2}=n\right\}, \\
d(1,1,2,2 ; n)=\#\left\{\left(n_{1}, n_{2}, n_{3}, n_{4}\right) \mid n_{1}, n_{2}, n_{3}, n_{4} \in \mathbb{N}, n_{1} n_{2} n_{3}^{2} n_{4}^{2}=n\right\},
\end{gathered}
$$

where $\mathbb{N}$ is the set of all natural numbers. Our results are:

TheOREM 1.

$$
\sum_{n \leq x} d(1,1,2 ; n)=\text { main terms }+O\left(x^{77 / 208+\varepsilon}\right) .
$$

\section{THEOREM 2.}

$$
\sum_{n \leq x} d(1,1,2,2 ; n)=\text { main terms }+O\left(x^{0.4+\varepsilon}\right) .
$$

Here $\varepsilon$ is an arbitrarily small given positive number, and $x$ is a large positive number. The exponent $77 / 208=0.3701 \ldots$ of Theorem 1 improves the corresponding exponent $3 / 8=0.375$ of Schmidt [10], and the exponent 0.4 of Theorem 2 improves the exponent $45 / 109=0.412 \ldots$ of Menzer and Seibold [9]. The connection of these divisor problems with the distribution of certain quantities of finite Abelian groups was first established in Krätzel [2]. Let $\tau(G)$ be the number of direct factors of a finite Abelian group $G$, and $t(G)$ be the number of unitary factors of $G$, and

$$
T(x)=\sum \tau(G), \quad T^{*}(x)=\sum t(G),
$$

where the summations are over all Abelian groups of order not exceeding $x$. Then from the arguments of [2] we get

Corollary 1. $T(x)=$ main terms $+O\left(x^{0.4+2 \varepsilon}\right)$.

Corollary 2. $T^{*}(x)=$ main terms $+O\left(x^{77 / 208+2 \varepsilon}\right)$.

After certain reductions our problems are connected with multiple exponential sums, which can be estimated as accurately as possible by means 
of the method given in the author's preceding papers [3]-[8] on similar divisor problems. A sharper estimate of Huxley [1] will also be appealed to in proving Theorem 1.

The author wishes to thank his colleagues, M. N. Huxley of Cardiff and E. Krätzel of Jena, for sending reprints of [1] and [2], and for their encouragement.

2. Proof of Theorem 1. Let

$$
S(a, b, c ; x)=\sum_{n^{a} m^{b+c} \leq x, n \leq m} \psi\left(\left(\frac{x}{n^{a} m^{b}}\right)^{1 / c}\right), \quad \psi(t)=t-[t]-1 / 2 .
$$

We have

LEMMA 1.

$$
\sum_{n \leq x} d(1,1,2 ; n)=\text { main terms }+\Delta(1,1,2 ; x),
$$

where

$$
\Delta(1,1,2 ; x)=-2 S(1,1,2 ; x)-2 S(1,2,1 ; x)-2 S(2,1,1 ; x)+O\left(x^{1 / 4}\right) .
$$

Proof. This is Lemma 5 of [2]. The expression for $\Delta(1,1,2 ; x)$ comes from a paper of Vogts (cf. Lemma 3 of Krätzel [2]).

For any permutation $(a, b, c)$ of $(1,1,2)$, it suffices for us to consider $S(M, N ; x)$, where $M$ and $N$ are integers with $2 M \geq N, M^{b+c} N^{a} \leq x$,

$$
S(M, N ; x):=S_{a, b, c}(M, N ; x)=\sum_{(m, n) \in D} \psi\left(\left(\frac{x}{n^{a} m^{b}}\right)^{1 / c}\right), \quad M N>x^{0.35},
$$

and $D:=D(M, N)=\left\{(m, n) \mid m \sim M, n \sim N, m^{b+c} n^{a} \leq x, n \leq m\right\}$. Throughout this paper, we use $r \sim R$ and $r \cong R$ to mean $1 \leq r / R<2$ and $C_{1} \leq r / R \leq C_{2}$, respectively; $C_{i}(i=1,2,3, \ldots)$ will be some absolute constants. In order to introduce exponential sums we apply the familiar Fourier expansion treatment of the function $\psi(t)$; thus for a parameter $K \geq 100$, we get, as on p. 266 of [3], the following estimate:

$$
\begin{aligned}
& (\ln x)^{-1} S(M, N ; x) \\
& \ll M N K^{-1}+\sum_{1 \leq h \leq K^{2}} \min \left(1 / h, K / h^{2}\right)\left|\sum_{(m, n) \in D} e(f(h, m, n))\right|,
\end{aligned}
$$

where

$$
f(h, m, n)=h\left(\frac{x}{n^{a} m^{b}}\right)^{1 / c} .
$$


Thus for some $H \in\left[1, K^{2}\right]$ we have

$$
x^{-\varepsilon} S(M, N ; x) \ll M N K^{-1}+\min (1, K / H) \Phi(H, M, N),
$$

where

$$
\begin{aligned}
\Phi(H, M, N) & :=\Phi_{a, b, c}(H, M, N) \\
& =H^{-1} \sum_{h \sim H}\left|\sum_{(m, n) \in D} e(f(h, m, n))\right| .
\end{aligned}
$$

Similarly to (1) and (2) of [7] we get (we have omitted the routine details for simplicity)

$$
\begin{aligned}
& \Phi(H, M, N) \\
& \ll H^{-1}\left(M^{2}(H F)^{-1}\right)^{1 / 2} \sum_{h \sim H}\left|\sum_{(u, n) \in D_{1}} P(u) Q(n) e\left(g_{1}(h, u, n)\right)\right| \\
& \quad+(H F)^{1 / 2}+x^{1 / 3}
\end{aligned}
$$

and

$$
\begin{aligned}
\text { (4) } \Phi(H, M, N) \ll & M N\left(H^{2} F\right)^{-1} \sum_{h \sim H}\left|\sum_{(u, v) \in D_{2}} R(u) S(v) e\left(g_{2}(h, u, v)\right)\right| \\
& +(H F)^{1 / 2}+x^{1 / 3}
\end{aligned}
$$

where $F=\left(x M^{-b} N^{-a}\right)^{1 / c}, D_{1}$ and $D_{2}$ are subsets of $\{(u, n) \mid u / U \in$ $\left.\left[C_{1}, C_{2}\right], n \in[N, 2 N)\right\}$ and $\left\{(u, v) \mid u / U \in\left[C_{3}, C_{4}\right], v / V \in\left[C_{5}, C_{6}\right]\right\}$, respectively, both are embraced by $O(1)$ algebraic curves, $P(\cdot), Q(\cdot), R(\cdot), S(\cdot)$ are monomials of the form $A t^{\alpha}$, with $A$ being the number independent of variables, and $\alpha$ being a rational, and

$$
\begin{gathered}
|P(\cdot)|,|Q(\cdot)|,|R(\cdot)|,|S(\cdot)| \leq 1 ; \\
g_{1}(h, u, n)=C_{7}\left(x h^{c} u^{b} n^{-a}\right)^{1 /(c+b)}, \quad g_{2}(h, u, v)=C_{8}\left(x h^{c} u^{b} v^{a}\right)^{1 / 4} ; \\
U=H F M^{-1}, \quad V=H F N^{-1} .
\end{gathered}
$$

We can apply Theorem 3 of [4] to estimate the triple exponential sum in (3), with the choice $(h, x, y)=(h, u, n)$; this yields

$$
\begin{aligned}
x^{-\varepsilon} \Phi(H, M, N) \ll & \sqrt[22]{H^{8} F^{11} M^{3} N^{13}}+(H F)^{1 / 2} N^{5 / 8}+\sqrt[16]{H^{4} F^{4} N^{17}} \\
& +\sqrt[32]{H^{8} F^{11} M^{3} N^{28}}+\sqrt[32]{H^{13} F^{16} M^{3} N^{18}} \\
& +\sqrt[4]{F M N^{4}}+\sqrt[4]{H F^{2} M N^{2}}+x^{1 / 3}
\end{aligned}
$$

By putting the estimate (5) into (1) and choosing $K \in[0, x]$ optimally via Lemma 2 of [3], we get 
LEMMA 2.

$$
\begin{aligned}
x^{-2 \varepsilon} S(M, N ; x) \ll & \sqrt[30]{F^{11} M^{11} N^{21}}+\sqrt[24]{F^{8} M^{8} N^{18}}+\sqrt[20]{F^{4} M^{4} N^{21}} \\
& +\sqrt[40]{F^{11} M^{11} N^{36}}+\sqrt[45]{F^{16} M^{16} N^{31}}+\sqrt[5]{F^{2} M^{2} N^{3}} \\
& +\left(F M N^{4}\right)^{1 / 4}+x^{1 / 3} .
\end{aligned}
$$

Since $(a, b, c)$ is a permutation of $(1,1,2), M \gg N$ and $M^{b+c} N^{a} \leq x$, we have $F \ll x\left(M N^{2}\right)^{-1}$ and $N \ll x^{1 / 4}$, and thus by Lemma 2 we get

$$
x^{-2 \varepsilon} S(M, N ; x) \ll \sqrt[5]{x^{2} N^{-1}}+\left(x N^{2}\right)^{1 / 4}+x^{0.36} .
$$

We now use Huxley's results, which are better than those which can be deduced from [5]. By Theorem 4 of [1], for $(a, b, c)=(1,1,2)$ we have

$$
x^{-\varepsilon} S(M, N ; x) \ll N\left(\frac{M x}{N}\right)^{23 / 146} \ll\left(x^{46} N^{123}\right)^{1 / 219} \ll x^{0.36} ;
$$

for $(a, b, c)=(2,1,1)$ or $(1,2,1)$ we have

$$
x^{-\varepsilon} S(M, N ; x) \ll N\left(x N^{-2}\right)^{23 / 73}=\left(x^{23} N^{27}\right)^{1 / 73} .
$$

From (6)-(8) we get

$$
\text { (9) } \begin{aligned}
x^{-2 \varepsilon} S(M, N ; x) & \ll\left(x N^{2}\right)^{1 / 4}+\min \left(\left(x^{23} N^{27}\right)^{1 / 73}, \sqrt[5]{x^{2} N^{-1}}\right)+x^{0.36} \\
& \ll\left(x N^{2}\right)^{1 / 4}+x^{77 / 208} .
\end{aligned}
$$

To remove the term $\left(x N^{2}\right)^{1 / 4}$ we use Kolesnik's method.

Lemma 3. Let $f(x, y)$ be an algebraic function in the rectangle $D_{0}=$ $\{(x, y) \mid x \sim X, y \sim Y\}$ with $f(x, y) \sim_{\Delta} A x^{\alpha} y^{\beta}$ throughout $D_{0}$, and let $D$ be a subdomain of $D_{0}$ bounded by $O(1)$ algebraic curves. Suppose that $X \gg Y$, $N=X Y, A>0, F=A X^{\alpha} Y^{\beta}, \alpha \beta(\alpha+\beta-1)(\alpha+\beta-2) \neq 0,0<\Delta<\varepsilon_{0}$, where $\varepsilon_{0}$ is a small number depending at most on $\alpha$ and $\beta$. Then

$$
\begin{aligned}
\sum_{(x, y) \in D} e(f(x, y)) \ll_{\varepsilon, \alpha, \beta} & \left(\sqrt[6]{F^{2} N^{3}}+N^{5 / 6}+\sqrt[10]{\Delta^{4} Y^{4} F^{2} N^{5}}\right. \\
& +\sqrt[8]{F^{-1} X^{-1} N^{8}}+N F^{-1 / 4} \\
& \left.+\sqrt[4]{\Delta X^{-1} N^{4}}+N Y^{-1 / 2}\right)(N F)^{\varepsilon / 2} .
\end{aligned}
$$

Proof. See Lemma 1.5 of [6]. This result is due to Kolesnik.

By Cauchy's inequality and Weyl's inequality (cf. Lemma 3 of [3]), after a partial summation removing the smooth coefficient $S(v)$ together with an appeal to Lemma 1 of [3] relaxing the range of $v$, we get for the double summation over $(u, v)$ in $(4)$ the following estimate:

$$
x^{-\varepsilon}\left|\sum_{(u, v) \in D_{2}} R(u) S(v) e\left(g_{2}(u, v)\right)\right|^{2} \ll \frac{(U V)^{2}}{Q}+\frac{U V}{Q} \sum_{1 \leq q \leq Q}\left|\sum_{(u, v) \in D(q)} e\left(g_{3}\right)\right|,
$$


where

$$
\begin{gathered}
D(q)=\left\{(u, v) \mid u \in\left[C_{3} U, C_{4} U\right], v \in\left[C_{5} V, C_{6} V\right],(v+q) \in\left[C_{5} V, C_{6} V\right]\right\}, \\
g_{3}=g_{3}(h, u, v, q)=g_{2}(h, u, v+q)-g_{2}(h, u, v), \\
Q=\min \left(V(\ln x)^{-1}, \sqrt[8]{(H F)^{-2} U^{3} V^{5}} .\right.
\end{gathered}
$$

If $Q \ll 1$ the above inequality holds obviously. Assume that $Q \gg 1$. We apply Lemma 3 to the inner double exponential sum over $(u, v)$, with the choice $X \cong V, Y \cong U, \Delta=q / V, F \cong H F q / V$, to obtain

$$
\begin{aligned}
x^{-\varepsilon} \sum_{(u, v) \in D(q)} e\left(g_{3}\right) \ll & \sqrt[6]{(H F)^{2} q^{2} U^{3} V}+(U V)^{5 / 6}+\sqrt[10]{(H F)^{2} q^{6} U^{9} V^{-1}} \\
& +\sqrt[8]{(H F)^{-1} q^{-1} U^{8} V^{8}}+(H F q)^{-1 / 4} U V^{5 / 4} \\
& +\sqrt[4]{q U^{4} V^{2}}+V U^{1 / 2}
\end{aligned}
$$

and so

$$
\begin{aligned}
x^{-2 \varepsilon} & \sum_{(u, v) \in D_{2}} R(u) S(v) e\left(g_{2}(u, v)\right) \\
\ll & (U V)^{11 / 12}+V U^{3 / 4}+\sqrt[16]{(H F)^{2} U^{13} V^{11}}+\sqrt[80]{(H F)^{2} U^{85} V^{51}} \\
& +\sqrt[64]{(H F)^{-2} U^{67} V^{53}}+\sqrt[16]{(H F)^{-1} U^{16} V^{15}} \\
& +\sqrt[128]{(H F)^{-6} U^{125} V^{123}}+U V(H F)^{-1 / 8}+\sqrt[64]{(H F)^{-6} U^{61} V^{67}} .
\end{aligned}
$$

By substituting (10) in (4) we get

$$
\begin{aligned}
x^{-2 \varepsilon} \Phi(H, M, N) \ll & \sqrt[12]{(H F)^{10} M N}+\sqrt[16]{(H F)^{10} M^{3} N^{5}} \\
& +\sqrt[4]{(H F)^{3} M}+\sqrt[80]{(H F)^{58} M^{-5} N^{29}} \\
& +\sqrt[64]{(H F)^{54} M^{-3} N^{11}}+\sqrt[16]{(H F)^{14} N} \\
& +\sqrt[128]{(H F)^{114} M^{3} N^{5}}+(H F)^{7 / 8} \\
& +\sqrt[64]{(H F)^{58} M^{3} N^{-3}}+x^{1 / 3}
\end{aligned}
$$

We put the estimate of (11) in (1) and choose $K \in[0, x]$ optimally via Lemma 2 of [3] to get

$$
\begin{aligned}
x^{-3 \varepsilon} S(M, N ; x) \ll & \sqrt[22]{F^{10}(M N)^{11}}+\sqrt[26]{F^{10} M^{13} N^{15}} \\
& +\sqrt[7]{F^{3} M^{4} N^{3}}+\sqrt[138]{F^{58} M^{53} N^{87}} \\
& +\sqrt[118]{F^{54} M^{51} N^{65}}+\sqrt[30]{F^{14} M^{14} N^{15}} \\
& +\sqrt[242]{F^{114} M^{117} N^{119}}+\sqrt[15]{(F M N)^{7}} \\
& +\sqrt[122]{F^{58} M^{61} N^{55}}+x^{1 / 3}
\end{aligned}
$$




$$
\begin{aligned}
\ll & \sqrt[22]{x^{10} M N^{-9}}+\sqrt[26]{x^{10} M^{3} N^{-5}} \\
& +\sqrt[7]{x^{3} M N^{-3}}+\sqrt[138]{x^{58} M^{-5} N^{-29}}+x^{1 / 3} \\
& +\sqrt[118]{x^{54} M^{-3} N^{-43}}+\sqrt[30]{x^{14} N^{-13}} \\
& +\sqrt[242]{x^{114} M^{3} N^{-109}}+\sqrt[15]{x^{7} N^{-7}} \\
& +\sqrt[122]{x^{58} M^{3} N^{-61}}
\end{aligned}
$$

by using the fact that $F \ll x M^{-1} N^{-2}$. From (9) and (12) we deduce that

$$
x^{-3 \varepsilon} S(M, N ; x) \ll \sum_{1 \leq i \leq 9} E_{i}+x^{77 / 208},
$$

where (note that $M N \ll x^{1 / 2}$ always holds)

$$
\begin{aligned}
E_{1} & =\min \left(\left(x N^{2}\right)^{1 / 4}, \sqrt[22]{x^{10} M N^{-9}}\right) \leq\left(x^{15} M N\right)^{1 / 42} \ll x^{31 / 84}<x^{0.37}, \\
E_{2} & =\min \left(\left(x N^{2}\right)^{1 / 4}, \sqrt[26]{x^{10} M^{3} N^{-5}}\right) \leq\left(x^{14}(M N)^{3}\right)^{1 / 42} \\
& \ll x^{31 / 84}<x^{0.37}, \\
E_{3} & =\min \left(\left(x N^{2}\right)^{1 / 4}, \sqrt[7]{x^{3} M N^{-3}}\right) \leq\left(x^{5} M N\right)^{1 / 15} \ll x^{11 / 30}<x^{0.37}, \\
E_{4} & =\min \left(\left(x N^{2}\right)^{1 / 4}, \sqrt[138]{x^{58} N^{-34}}\right) \leq x^{75 / 206}<x^{0.37}, \\
E_{5} & =\min \left(\left(x N^{2}\right)^{1 / 4}, \sqrt[118]{x^{54} N^{-46}}\right) \leq x^{77 / 210} \\
E_{6} & =\min \left(\left(x N^{2}\right)^{1 / 4}, \sqrt[30]{x^{14} N^{-13}}\right) \leq x^{41 / 112}<x^{0.37}, \\
E_{7} & =\min \left(\left(x N^{2}\right)^{1 / 4}, \sqrt[242]{x^{114} M^{3} N^{-109}}\right) \leq\left(x^{170}(M N)^{3}\right)^{1 / 466} \\
\ll & \ll x^{343 / 932}<x^{0.37}, \\
E_{8} & =\min \left(\left(x N^{2}\right)^{1 / 4}, \sqrt[15]{x^{7} N^{-7}}\right) \leq x^{21 / 58}<x^{0.37}, \\
E_{9} & =\min \left(\left(x N^{2}\right)^{1 / 4}, \sqrt[122]{x^{58} M^{3} N^{-61}}\right) \leq\left(x^{90}(M N)^{3}\right)^{1 / 250} \\
& \ll x^{91.5 / 250}<x^{0.37},
\end{aligned}
$$

whence the required estimate follows.

3. Proof of Theorem 2. Let

$$
S(a, b, c, d ; x)=\sum_{n_{1}^{a} n_{2}^{b} n_{3}^{c+d} \leq x, 1 \leq n_{1}(\leq) n_{2} \leq n_{3}} \psi\left(\left(\frac{x}{n_{1}^{a} n_{2}^{b} n_{3}^{c}}\right)^{1 / d}\right),
$$

where $n_{1}(\leq) n_{2}$ means that $n_{1} \leq n_{2}$ for $(a, b)=\left(a_{i}, a_{j}\right)$ with $i<j$, and $n_{1}<n_{2}$ otherwise; here we have set $\left(a_{1}, a_{2}, a_{3}, a_{4}\right)=(1,1,2,2)$. Then we have 
LEMMA 4.

$$
\sum_{n \leq x} d(1,1,2,2 ; n)=\text { main terms }+\Delta(1,1,2,2 ; x),
$$

where

$$
\Delta(1,1,2,2 ; x)=-\sum_{(a, b, c, d)} S(a, b, c, d ; x)+O\left(x^{1 / 3}\right),
$$

and $(a, b, c, d)$ runs through all the ordered permutations of $(1,1,2,2)$.

Pr o of. The expression for the remainder $\Delta(1,1,2,2 ; x)$ is due to Vogts, see $[2]$.

In what follows we use the method presented in [8] for 4-dimensional exponential sums, but the details are much simpler here, and we omit many routine procedures. The reader is invited to consult [8]. It suffices for us to achieve an estimate of the type $S(a, b, c, d ; \boldsymbol{N}) \ll x^{0.4+4 \varepsilon}$, where $\boldsymbol{N}=$ $\left(N_{1}, N_{2}, N_{3}\right), N_{1}, N_{2}$ and $N_{3}$ are arbitrary positive integers with

$$
N_{1} \ll N_{2} \ll N_{3}, \quad N_{1}^{a} N_{2}^{b} N_{3}^{c+d} \leq x, \quad N_{1} N_{2} N_{3}>x^{0.37},
$$

$(a, b, c, d)$ is any permutation of $(1,1,2,2)$, and

$$
S(a, b, c, d ; \boldsymbol{N})=\sum^{*} \psi\left(\left(\frac{x}{n_{1}^{a} n_{2}^{b} n_{3}^{c}}\right)^{1 / d}\right),
$$

where $\sum^{*}$ denotes summation over lattice points $\left(n_{1}, n_{2}, n_{3}\right)$ with

$$
n_{1}^{a} n_{2}^{b} n_{3}^{c+d} \leq x, \quad 1 \leq n_{1}(\leq) n_{2} \leq n_{3}, \quad N_{v} \leq n_{v}<2 N_{v} \quad(v=1,2,3) .
$$

Let $G=\left(x N_{1}^{-a} N_{2}^{-b} N_{3}^{-c}\right)^{1 / d}$. As in (12) of [8], we can deduce

LEMMA 5.

$$
\begin{aligned}
x^{-2 \varepsilon} S(a, b, c, d ; \boldsymbol{N}) \ll & \sqrt[30]{G^{11} N_{1}^{30} N_{2}^{21} N_{3}^{11}}+\sqrt[24]{\left(G N_{3}\right)^{8} N_{2}^{18} N_{1}^{24}} \\
& +\sqrt[20]{\left(G N_{3}\right)^{4} N_{2}^{21} N_{1}^{20}}+\sqrt[40]{\left(G N_{3}\right)^{11} N_{2}^{36} N_{1}^{40}} \\
& +\sqrt[45]{\left(G N_{3}\right)^{16} N_{2}^{31} N_{1}^{45}}+\sqrt[5]{\left(G N_{3}\right)^{2} N_{2}^{3} N_{1}^{5}} \\
& +\sqrt[4]{G N_{3} N_{2}^{4} N_{1}^{4}}+x^{13 / 36}
\end{aligned}
$$

Similarly to (17) of [8], we also have

Lemma 6. $x^{-3 \varepsilon} S(a, b, c, d ; \boldsymbol{N}) \ll\left(G N_{1} N_{2} N_{3}\right)^{1 / 2}+x^{13 / 36}$.

Note that the term $x^{13 / 36}$ comes from an application of Lemma 1 of [8] (see also Lemma 1.4 of [6]) to the variable $n_{3}$ together with an estimate for the resulting "extra" term $R\left(h, n_{1}, n_{2}\right)$ (involving the use of the exponent pair $(1 / 6,4 / 6))$. From (13) it is seen that $G \ll x\left(N_{1}^{2} N_{2}^{2} N_{3}\right)^{-1}$ and $N_{1} N_{2} N_{3} \ll x^{1 / 2}$. Thus Lemmas 5 and 6 give respectively (with $J=N_{1} N_{2}$ )

$$
\begin{aligned}
x^{-4 \varepsilon} S(a, b, c, d ; \boldsymbol{N}) \ll & \sqrt[30]{x^{11} J^{3.5}}+\sqrt[24]{x^{8} J^{5}}+\sqrt[20]{x^{4} J^{13}} \\
& +\sqrt[40]{x^{11} J^{16}}+\sqrt[4]{x J^{2}}+x^{0.4},
\end{aligned}
$$


and

$$
x^{-4 \varepsilon} S(a, b, c, d ; \boldsymbol{N}) \ll\left(x J^{-1}\right)^{1 / 2}+x^{13 / 36} .
$$

Now if $J \geq x^{0.2}$ then the required estimate follows from (15), and otherwise it follows from (14). Thus Theorem 2 has been verified.

\section{References}

[1] M. N. Huxley, Exponential sums and lattice points II, Proc. London Math. Soc. 66 (1993), 279-301.

[2] E. Krätzel, On the average number of direct factors of a finite Abelian group, Acta Arith. 51 (1988), 369-379.

[3] H.-Q. Liu, On the number of abelian groups of a given order, ibid. 59 (1991), 261-277.

[4] - On the number of abelian groups of a given order (supplement), ibid. 64 (1993), 285-296.

[5] - , The number of squarefull numbers in an interval, ibid. 64 (1993), 129-149.

[6] - , The greatest prime factor of the integers in an interval, ibid. 65 (1993), 301-328.

[7] —, The number of cube-full numbers in an interval, ibid. 67 (1994), 1-12.

[8] - , The distribution of 4-full numbers, ibid. 67 (1994), 165-176.

[9] H. Menzer and R. Seibold, On the average number of direct factors of a finite Abelian group, Monatsh. Math. 110 (1990), 63-72.

[10] P. G. Schmidt, Zur Anzahl unitärer Faktoren abelscher Gruppen, Acta Arith. 64 (1993), 237-248.

206-10, BAO GUO ST.

HARBIN, 150066

CHINA 